\title{
MORPHOLOGY OF EARLY CHOROIDAL NEOVASCULARISATION IN AGE-RELATED MACULAR DEGENERATION: CORRELATION WITH ACTIVITY
}

\author{
J. P. SARKS ${ }^{1}$, S. H. SARKS ${ }^{1}$ and M. C. KILLINGSWORTH ${ }^{2}$ \\ Sydney, Australia
}

\begin{abstract}
SUMMARY
The purpose of this study was to describe the morphology of early choroidal neovascularisation (CNV) and compare the findings in two patients, in the first of whom the vessels appeared inactive and in the second progressive. Changes common to both included a diffuse deposition of membrane coils external to the basement membrane (BsM) of the retinal pigment epithelium (RPE), and macrophages and foreign body giant cells beneath thinned segments of Bruch's membrane (BrM). In the first patient small activated vessels surrounded by enlarged pericytes were found in the choroid beneath these areas and pursued a convoluted course, bulging into or through BrM, but without spreading in the sub-RPE space. This choroidal phase of CNV may be common and unrecognised. In the second patient there was spread of CNV in the sub-RPE space with extravasation of red cells and fibrin. The tips leaking fibrin were covered by a thick BsM-like material and naked endothelial cells were not seen. Pericytes were absent here but were observed at the non-leaking edge. The difference in activity of $\mathrm{CNV}$ in the two patients appeared to correlate with the quantity of membranous debris present.
\end{abstract}

Choroidal new vessels, often clinically unsuspected, are found commonly on histological examination of post-mortem eyes suffering from age-related maculopathy $(A R M) .^{1,2}$ In one study ${ }^{3}$ they were present even in about one-third of eyes with geographic atrophy of the retinal pigment epithelium (RPE), being detected in 15 of 46 eyes examined although

From: ${ }^{1}$ Department of Ophthalmology, University of New South Wales and Prince of Wales Medical Research Institute, Sydney, Australia; ${ }^{2}$ Electron Microscope Unit, South Western Area Pathology Services, Liverpool, NSW, Australia.

Correspondence to: Dr John Sarks, 15 Parnell Street, Strathfield, NSW, Australia 2135. mostly remaining confined. Since in ARM the early new vessels grow initially in the cleavage plane created by a diffuse layer of debris external to the basement membrane (BsM) of the RPE, disappearance of this sub-RPE space as atrophy develops may be the factor limiting their spread. On the other hand, active choroidal neovascularisation (CNV) is known to be related to soft confluent drusen ${ }^{4,5}$ which open this cleavage plane. The progress of $\mathrm{CNV}$ is therefore influenced by the nature and quantity of debris present.

In a recent report ${ }^{6}$ two phases of $\mathrm{CNV}$ in humans were described: an initial 'low-turnover' choroidal phase and a subsequent 'high-turnover' phase in the sub-RPE space characterised by extensive endothelial cell proliferation and migration. Attempts to prevent overt CNV by applying gentle photocoagulation to cause soft drusen to resorb therefore need to take into account the possible presence of the clinically unrecognised choroidal phase of CNV. In the present study the morphology of unsuspected early CNV which failed to spread in the sub-RPE space is contrasted with an early but active choroidal neovascular membrane (CNVM), still confined beneath the RPE, which leaked fibrinous exudate. The aim was to correlate the structure of these early neovascular proliferations with their apparent activity and, because of the lack of a natural animal model, draw comparisons with in vitro and in vivo experimental models of neovascularisation. Attention is drawn to the initial choroidal phase of $\mathrm{CNV}$ and to the difficulty in its clinical recognition.

\section{MATERIALS AND METHODS}

The light and electron microscopic findings are described in three eyes of two patients demonstrating early CNV. The first patient was an 83-year-old man last examined 1 year before death. Vision had been 
6/6 in each eye and his fundi had shown only a moderate number of small hard and soft drusen without pigment changes. Fluorescein angiography had revealed no evidence of CNV. The left fundus has been illustrated previously.

The second patient demonstrated more soft drusen. Over a 10-year period of clinical documentation there had been an increase in the number and confluence of the drusen, which measured up to $250 \mu \mathrm{m}$, the heaviest concentration occurring at the temporal edge of the fovea. The fundus picture and the morphology of the drusen have been reported previously. ${ }^{8}$ A fluorescein angiogram could not be obtained due to the patient's dementia. On his last visit, 2 months before death at the age of 75 years, the right eye was noted to have developed a haemorrhagic disciform scar and this eye is not described further. In the left eye vision was $6 / 36$ and the fundus was partly obscured by lens opacities. A CNVM which was found on pathological examination but had not been noted clinically is the subject of this report.

The eyes were prepared for electron microscopy according to techniques previously described. ${ }^{6}$ In the first patient sections were examined from blocks taken from the fovea of both eyes. In case 2 the CNVM was found extending diagonally across the fovea from upper nasal to lower temporal sides. The area examined measured $1950 \mu \mathrm{m}$ in length with a maximum width of $1050 \mu \mathrm{m}$ and included the upper, temporal and lower edges of the membrane, but nasally the membrane extended beyond the cut edge of the block. The origin of the new vessels from the choroid was not found.

\section{RESULTS \\ Case 1. Example of Initial Choroidal Phase of CNV}

The findings in both eyes of case 1 were similar. The RPE remained as an intact layer but was moderately enlarged due to the accumulation of complex lipofuscin granules. Other evidence of degeneration was the presence of diffuse deposits, comprising the basal laminar deposit (BLD) between the plasma cell membrane and the BsM of the RPE, and a layer of membrane coils lying between the RPE BsM and the inner collagenous zone of Bruch's membrane (BrM). A moderate number of small hard drusen were present, appearing as electron-dense hemispherical elevations on BrM (Figs. 1, 2). The membranous debris formed a shallow layer (Fig. 2) but was occasionally raised into larger mounds referred to as soft membranous drusen. ${ }^{8}$ Combinations of hard and membranous drusen were also found, the membrane coils separating the amorphous hard drusen material from the inner collagenous zone of BrM.

Macrophages and foreign body giant cells were found applied to the outer surface of $\mathrm{BrM}$, most frequently beneath small hard drusen (Fig. 2). Segments of thinning of the outer collagenous zone of $\mathrm{BrM}$ were present, measuring up to $100 \mu \mathrm{m}$ wide and with edges bevelled from below. Activation of the underlying pre-existing capillaries was also noted, the normally sparse pericytes demonstrating hypertrophy with a voluminous watery cytoplasm, subplasmalemmal densifications and cytoskeletal filaments (Figs. 1, 3). Although maintaining cellular contact with endothelial cells, the pericytes extended blunt projections into the choroidal stroma. The BsM between the endothelial cells and the pericytes was commonly thickened and pointed processes from the endothelial cells protruded into this material (Fig. 3), although only a few short slender endothelial cell processes actually breached the BsM. Evidence of activation of choroidal endothelial cells was also present, the cells demonstrating abundant organelles, an enlarged nucleus and the formation of long narrow pseudopodia partly enveloping the pericytes, ${ }^{6}$ but the vessels were always surrounded by dilated pericytes.

In at least four areas in each eye these activated vessels demonstrated varying degrees of penetration of $\mathrm{BrM}$. In one area a vessel displayed only the choroidal phase of angiogenesis (Fig. 4), a capillary surrounded by dilated pericyte processes extending about $20 \mu \mathrm{m}$ into the choroid and pursuing a convoluted course before becoming a postcapillary venule. Enlarged cells were noted also in the walls of the postcapillary venule but immunohistochemistry, which might have distinguished pericytes from myofibroblasts, ${ }^{9}$ was not performed. Adjacent choroidal capillaries were narrowed or absent, but it could not be determined whether the new vessel proliferation had occurred in a pre-existing focal area of hypoperfusion or whether the choriocapillary narrowing was secondary to altered haemodynamics following $\mathrm{CNV}$.

Greater penetration caused fragmentation and displacement of the inner collagenous layer (Fig. 1), while complete penetration of the collagenous zones resulted in elevation of the RPE (Fig. 5). However, serial sections through the discontinuities in $\mathrm{BrM}$ confirmed that these gaps then closed again and the vessels did not progress further. A narrow lumen was present in these sprouts and fenestrations were identified, but the endothelial cells remained surrounded by dilated pericytes. These early new vessels were quite small, being less than $100 \mu \mathrm{m}$ across.

In one area the vessels did extend for a short distance (about $100 \mu \mathrm{m}$ ) along the inner surface of $\mathrm{BrM}$, but they were surrounded by collagen and the underlying choroid also was more fibrotic. The pericytes appeared mature and these appeared to be inactive vessels. The lack of further progression in this patient may have been related to the presence of 


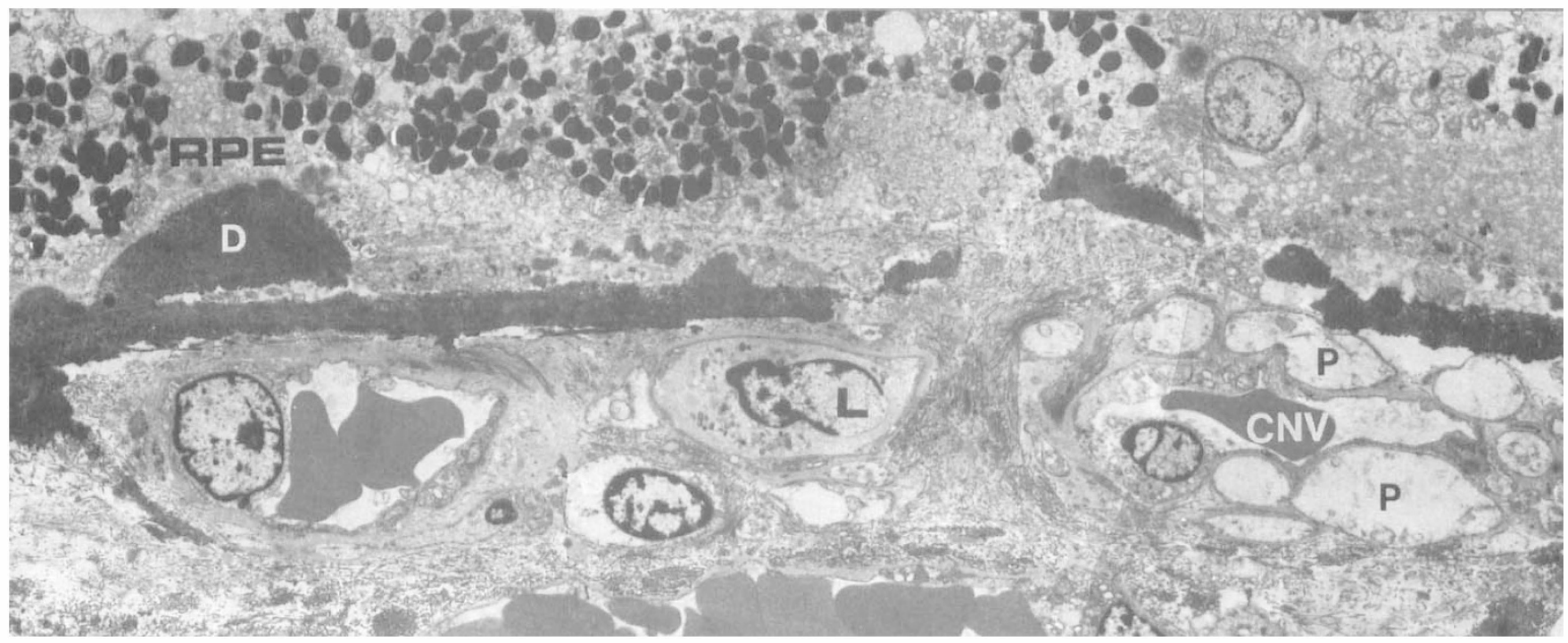

Fig. 1. Case 1, left eye. Electron micrograph showing, at right, an activated choroidal capillary (CNV) herniating into a disrupted Bruch's membrane. The vessel is surrounded by dilated pericyte processes $(P)$ embedded in thick basement membrane. Serial sections showed the gap closed again and the vessel did not progress further. A leucocyte $(L)$ is in the lumen of the adjacent capillary. Small amorphous hard drusen (D) lie on Bruch's membrane but there is minimal diffuse debris under the retinal pigment epithelium $(R P E) . \times 2210$.

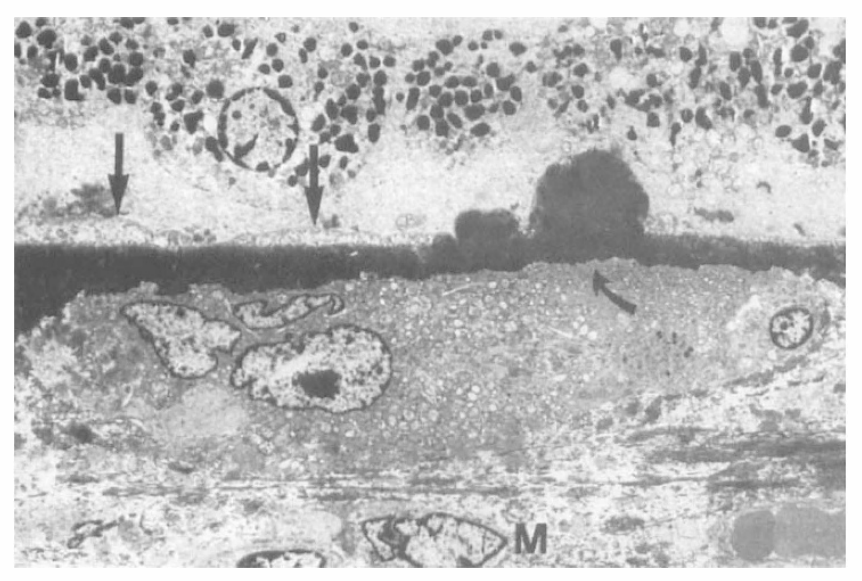

Fig. 2. Section close to Fig. 1, showing a foreign body giant cell closely applied to the outer surface of Bruch's membrane beneath two hard drusen. The outer collagenous zone appears thinned here (curved arrow). Drusen are less than one RPE cell in diameter and would not be visible clinically. A thin layer of membranous debris lies on the internal surface of Bruch's membrane, external to the basement membrane of the RPE (straight arrows). M, macrophage. $\times 1410$.

only a small amount of membranous debris under the adjacent RPE.

Case 2. Example of Sub-RPE Spread of Active CNV In this eye the CNVM spread in the sub-RPE space, in the plane created by soft drusen (Fig. 6). Hard drusen, on the other hand, had an anchoring effect on the RPE and the neovascular membrane had initially to grow around these deposits. A sensory retinal detachment was present over part of the membrane and swollen hyperpigmented cells were shed into the subsensory retinal space, but the RPE

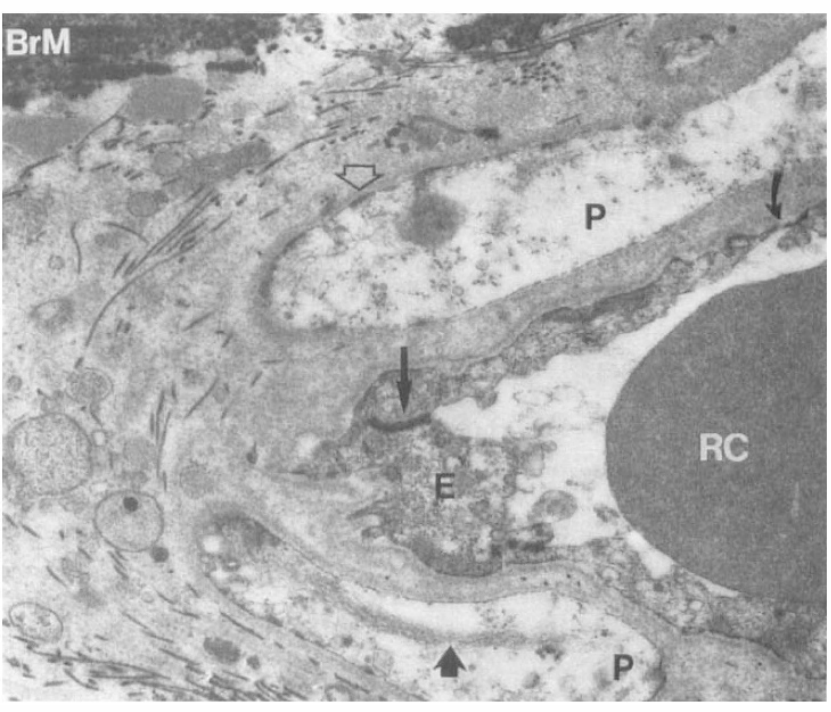

Fig. 3. Nearby section through the edge of the activated choroidal vessel shown in Fig. 1. Dilated pericyte processes $(P)$ demonstrate watery cytoplasm containing subplasmalemmal densifications (hollow arrowhead) and cytoskeletal filaments (filled arrowhead). Basement membrane of endothelial cell and pericyte is prominent. The endothelial cells $(E)$ extend pointed processes into the basement membrane between two pericyte processes. Straight arrow points to a tight junction between two endothelial cells which may be extending the lumen between the pericyte processes, but endothelial cells do not breach basement membrane. Fenestrations (curved arrow, upper right) were noted principally on the pigment epithelial side of the vessel, similar to those in normal choroidal capillaries. BrM, Bruch's membrane; $R C$, red cell in lumen. $\times 5220$.

remained intact and in contact with the membrane except just beyond the advancing tip where a small RPE detachment was present (Fig. 7). Strands of a fibrin-like material with a periodicity of $26 \mathrm{~nm}$ could 


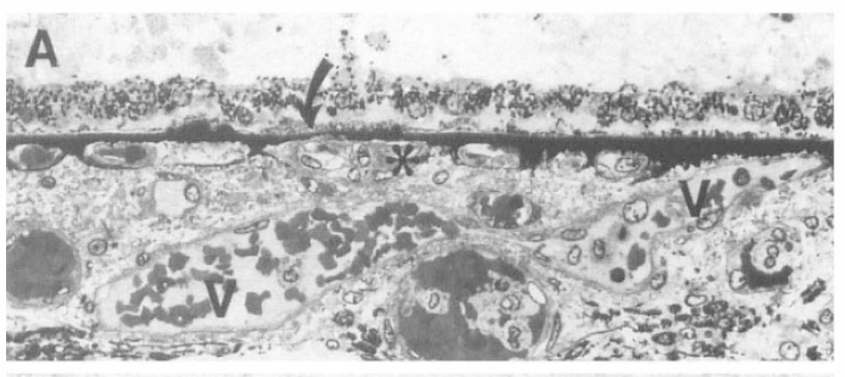

B

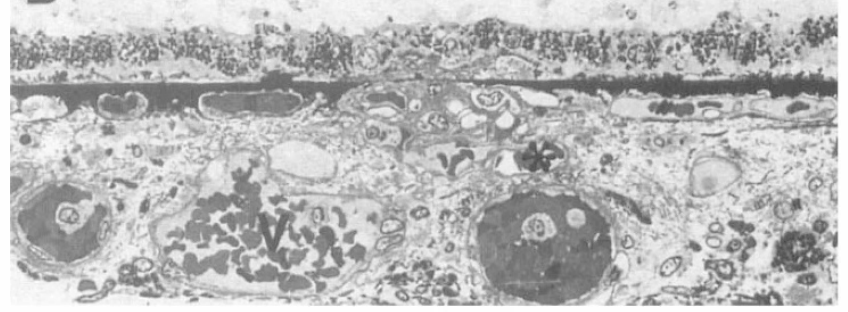

C
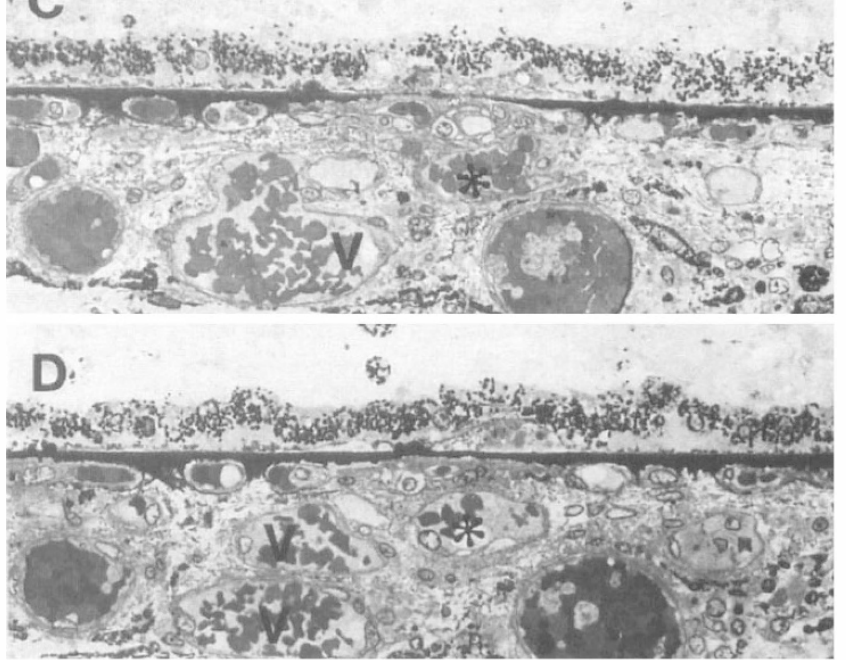

Fig. 4. Serial sections tracing the course of an intrachoroidal new vessel. (A) Bruch's membrane is thinned (curved arrow) in the vicinity of small drusen. Underlying capillary (asterisk) is surrounded by dilated pericytes. The choroidal vein $(V)$ drains the pre-existing capillary at upper right. (B) Bruch's membrane thins further. Underlying capillary pursues a convoluted course and becomes a postcapillary venule (asterisk). (C) Bruch's membrane remains very attenuated but is not penetrated. (D) Bruch's membrane thickens again. A dilated pericyte process is still evident in the wall of the postcapillary venule (asterisk) which joins the upper division of the choroidal vein. Methylene blue and basic fuchsin, $\times 210$.

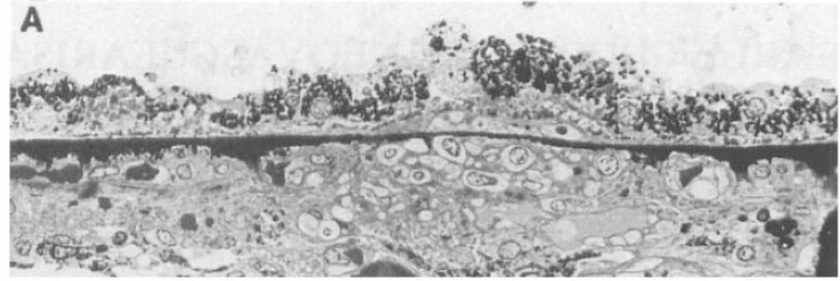

B
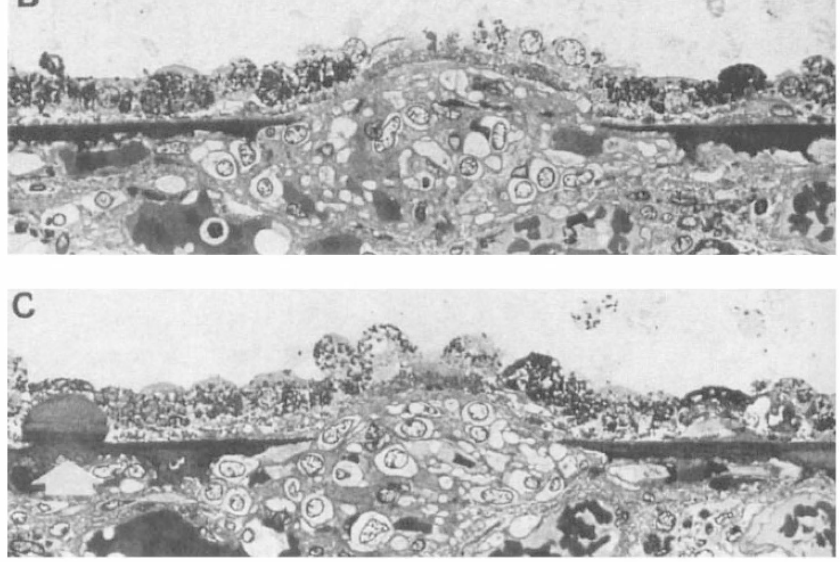

D

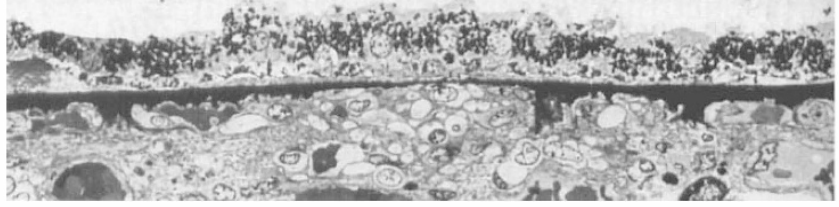

Fig. 5. Case 1, right eye. Serial sections showing an early new vessel bulging through collagenous layers of Bruch's membrane. $(A)$ and $(D)$ show the opening then closes. The vessel contains a narrow lumen lined by endothelium, but the predominant cells are enlarged pericytes which completely surround the vessel. A druse in (C) (white arrowhead), about two RPE cells across, represents the smallest druse visible clinically and indicates the small size of the vessel. Note the relative lack of debris beneath the RPE. Methylene blue and basic fuchsin, $\times 205$.

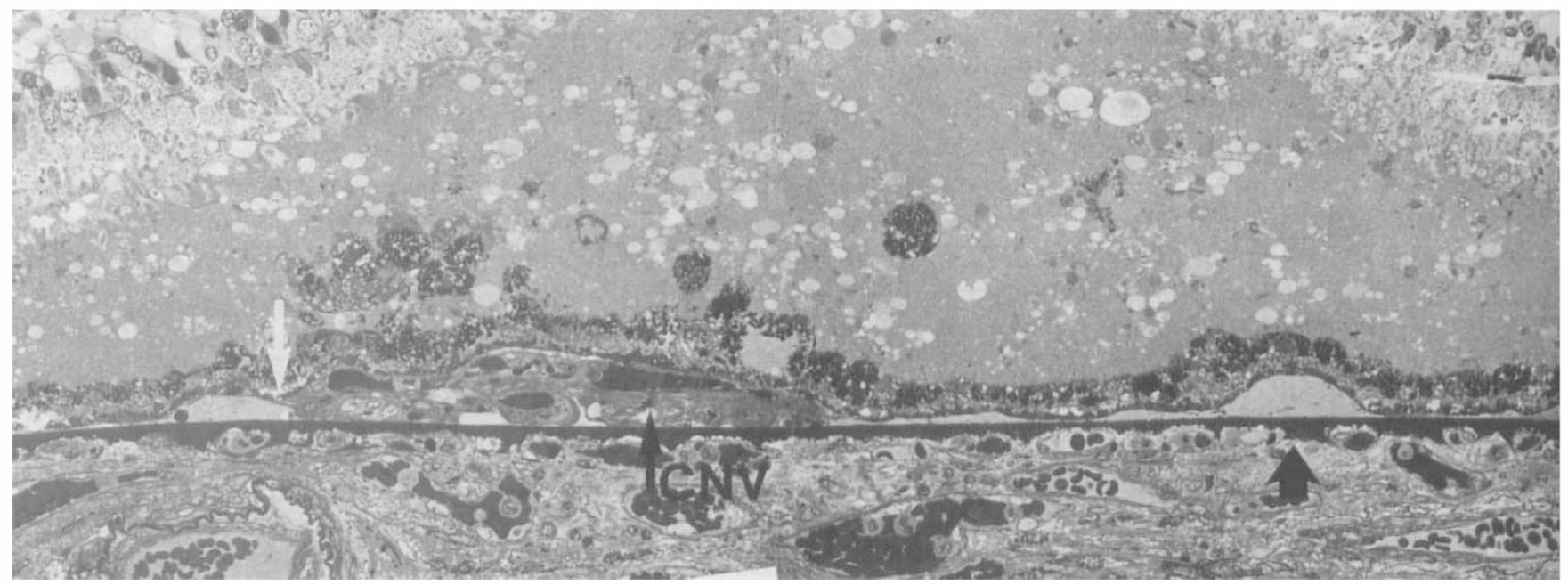

Fig. 6. Case 2. Semithin section through new vessel membrane (CNV) growing beneath the RPE in the plane of soft drusen (black arrowhead at right). Swollen hyperpigmented cells are shed into the fluid-filled subsensory retinal space. The apex of the membrane (white arrow) consists of a double layer of vessels. A small RPE detachment lying beyond the tip is magnified in Fig. 7. Methylene blue and basic fuchsin, $\times 2520$. 

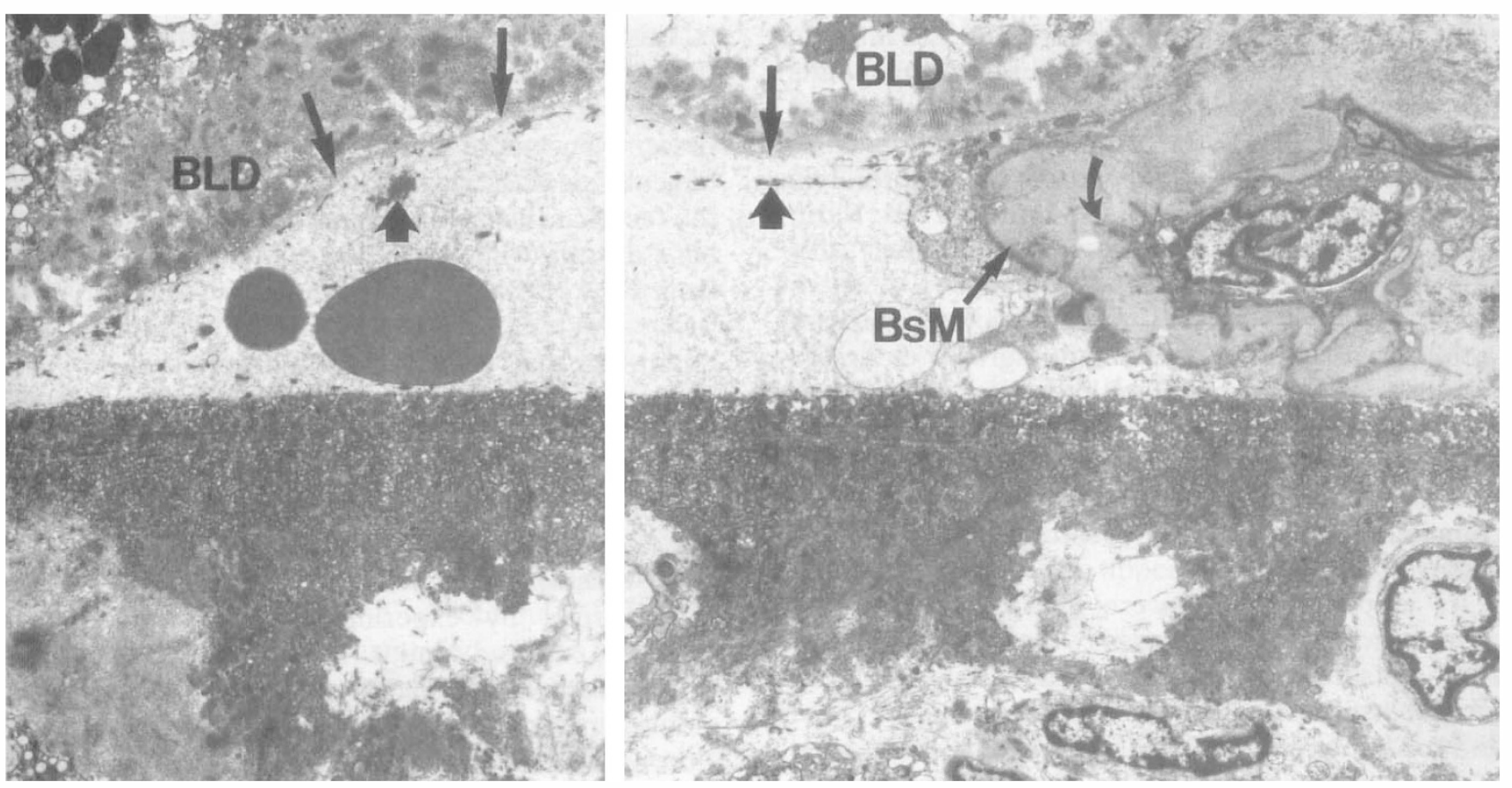

Fig. 7. Composite montage of the apex of the neovascular membrane indicated in Fig. 6. The tip of the vessel has a bulbous appearance due to a thick covering of basement membrane-like material (BsM) into which endothelial cells send processes (curved arrow). Note the absence of pericytes. RPE basement membrane (arrows) is separated from Bruch's membrane by a small RPE detachment containing red cells and strands of fibrin-like material (arrowheads) with a periodicity of $26 \mathrm{~nm}$. There is dropout of choroidal capillaries. BLD, basal laminar deposit. $\times 4160$.

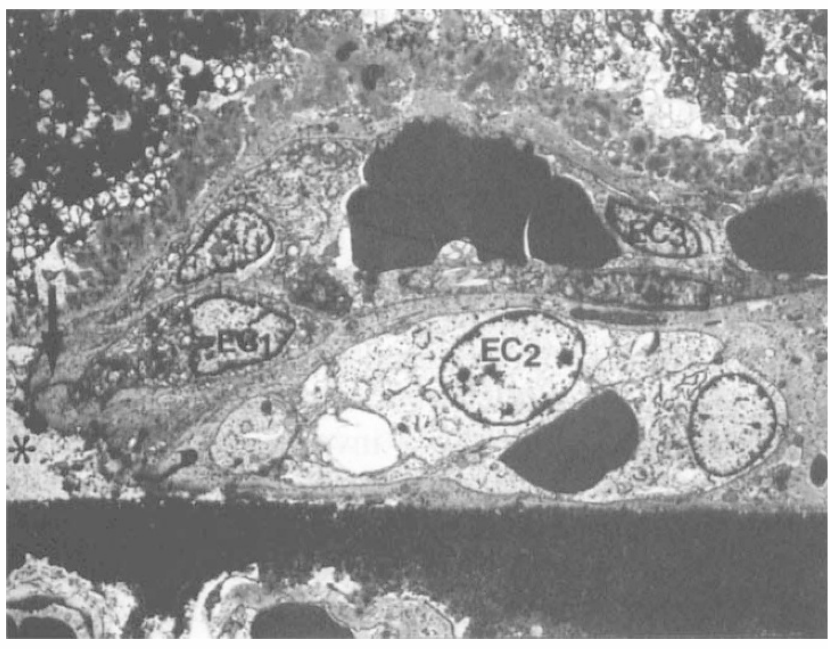

Fig. 8. Another view of a new vessel tip which ends blindly in a loop. Nuclei of three endothelial cell phenotypes are distinguished. The cell at the apex $\left(E C_{1}\right)$ has dense cytoplasm and appears to be migrating. It is extending processes or pseudopodia (arrow) into but not beyond the thick layer of basement membrane-like material. Pale endothelial cells with puffy cytoplasm $\left(E C_{2}\right)$ are consistent with the proliferative phase. More mature endothelial cells $\left(E C_{3}\right)$ lie behind the apex. Fibrin-containing exudate lies beyond the tip at the left (asterisk). $\times 1620$.

be traced from the vessel tip into the RPE detachment, in which there were also a few red cells.

The new vessel sprouts ended in blind loops. Sprouts with bulbous ends leaking fibrinous exudate protruded about $100 \mu \mathrm{m}$ in advance of the main membrane, their swollen appearance resulting from a

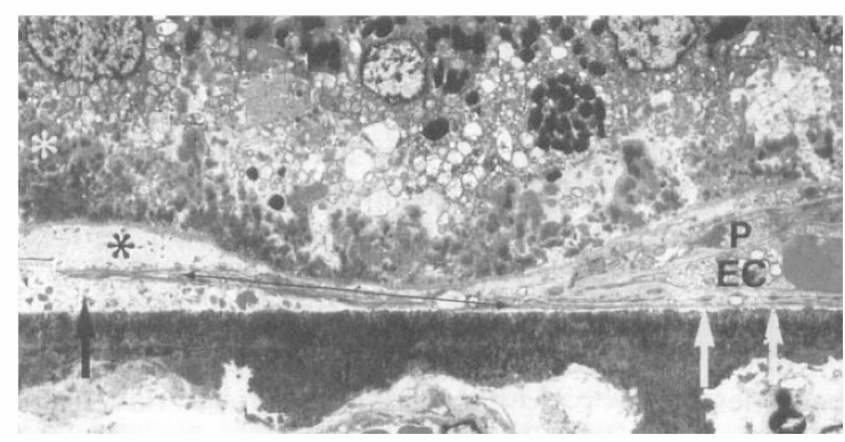

Fig. 9. Section showing spread of a new vessel in the plane of soft drusen. In this region endothelial cells $(E C)$ at the tip are partially surrounded by pericytes $(P)$. A cell process (thin double-ended arrow) is associated with fibrillar basement-membrane-like material and appears to arise from a myofibroblast-like pericyte showing subplasmalemmal densifications (white arrows). The cell process separates a small quantity of fibrin-containing exudate above (black asterisk) from soft drusen material below (black arrow), in which globules of amorphous material are mixed with membrane coils. Banded basal laminar deposit is prominent (white asterisk). $\times 1420$.

thick covering of amorphous BsM-like material (Figs. 7, 8). Pericytes were not observed at these tips and the BsM-like material was therefore secreted by the endothelial cells. Three endothelial cell phenotypes could be distinguished (Fig. 8). The cells at the apex had a dense cytoplasm and appeared 
to be migrating, extending slender processes into the BsM. Cell junctions were immature, fusion points rarely being observed. Behind the apex, puffy cells with pale cytoplasm containing rough endoplasmic reticulum appeared to represent a proliferative phase ${ }^{10}$ although mitoses were not observed. More proximally, mature fenestrated endothelial cells surrounded a well-developed lumen. Elsewhere along the edge of the CNVM pericyte processes with a condensed cytoplasm had migrated along the sprouts (Fig. 9) and the BsM-like material became a fibrillar early collagenous matrix. Where pericyte processes with prominent rough endoplasmic reticulum embedded in a thickened collagenous matrix completely surrounded the sprout, there was no leakage.

As in case 1 , there were multiple sites in the choroid where macrophages were applied to the outer surface of a thinned BrM. The BLD was a wellformed layer containing wide-spacing collagen (Figs. $7,9)$. Membranous debris was present in greater quantity than in case 1 and became the predominant constituent of soft drusen, although the drusen also contained small amorphous globules which were interpreted as broken-down hard drusen material (Fig. 9). Where soft drusen lay close to the tip of the CNVM serum was noted to enter several drusen (Fig. 9), whereas at the non-leaking edges there was only minimal drusen debris.

\section{DISCUSSION}

The earliest stages of CNV in ARM are difficult to study in the human. The morphological changes preceding $\mathrm{CNV}$ include the formation of diffuse deposits which are defined according to their relationship to the BsM of the RPE. The BLD lies between the RPE plasma membrane and BsM and becomes universal once degeneration develops. However, diffuse debris also appears on the external aspect of the BsM, termed the basal linear deposit by Green and Enger. ${ }^{11}$ This is more variable in quantity and on electron microscopy contains membrane coils which have the bilayered structure of lipids and which also become a major constituent of some soft drusen. ${ }^{8}$ It is at this stage that macrophages and foreign body giant cells are noted in increasing numbers in relation to the outer surface of BrM.7,12 The mobilisation of these cells is followed by the development of thinned segments in the overlying outer collagenous zone of BrM, within which one or more openings communicating with the potential sub-RPE space may then appear. Activation of choroidal capillaries was observed only beneath these thinned regions of $\mathrm{BrM}$, suggesting that angiogenic factors released by the macrophages may play a role.
This apparent erosion of BrM from the choroid ${ }^{7,13}$ therefore becomes apparent only when membranous debris and the soft drusen derived therefrom are present, but paradoxically it then often commences beneath small hard drusen. ${ }^{8}$ Possibly this is due to the fact that the RPE remains anchored for a time to hard drusen, while becoming increasingly separated from $\mathrm{BrM}$ elsewhere by the membranous debris. An ischaemic stimulus induced in the outer retina by this separation may cause the RPE to release diffusible angiogenic factors, vascular endothelial growth factor in particular being influenced by local oxygen concentrations ${ }^{14}$ these factors would reach the choroid in greatest concentration where the RPE remains attached to BrM. The link between ischaemia, macrophages and angiogenesis is further strengthened by experiments on bovine retinal explants cultured under hypoxic conditions, ${ }^{15}$ in which proliferating endothelial cells formed tubes only when macrophages were also present. However, although $\mathrm{CNV}$ is associated with choriocapillaris dropout in pathological specimens, ${ }^{16}$ cause and effect have not been established.

The early stages of angiogenesis in the choroid are difficult to reproduce also in animal models, in which attempts to promote $\mathrm{CNV}$ by laser ${ }^{17}$ first induce an acute inflammation not seen in ARM. Moreover, owing to the lack of drusen and diffuse deposits in these animals, there had been no prior dissociation of the sub-RPE space in which the vessels may spread, so that they immediately pass through the RPE and become subretinal. ${ }^{18}$ The early events thought to occur in the choroid have therefore been extrapolated from non-choroidal models of angiogenesis and in both in vitro neovascularisation ${ }^{19,20}$ and in vivo tumour-induced capillary proliferation in the rabbit cornea ${ }^{21}$ the initial stages involve the endothelial cells. In these experiments the endothelial cells extend cytoplasmic processes through the basement membrane but, since the origin of the new vessel sprouts occurs from preformed non-fenestrated vessels, the authors acknowledge that the same sequence may not occur in other forms of neovascularisation. Nevertheless, the endothelial cell is capable of making a capillary tube on its own ${ }^{19}$ and it has been suggested that the pericyte may not be involved in the initial vascular sprout and lumen formation, ${ }^{22}$ although it may play a secondary role in controlling capillary proliferation. ${ }^{23}$

In case 1, however, hypertrophy of pericytes of pre-existing capillaries and postcapillary venules was the most conspicuous response in the choroid. Migration of these activated pericytes away from the endothelial cells may even be the first event, thereby releasing the endothelial cells from contact inhibition. ${ }^{24}$ In the present study activation of choroidal endothelial cells was also observed, how- 
ever, so that it is possible that an initial endothelial cell response did take place in the choroid but became confined by proliferation of pericytes. It therefore cannot be concluded that the small pericyte-enshrouded vessels seen in case 1 were the precursor of the active CNVM in case 2 .

The earliest new vessels may even be entirely intrachoroidal $^{6}$ but, even if they bulge into BrM, such vessels may be so small that they may be difficult to distinguish on fluorescein angiography from brightly staining drusen. The presence of fenestrations implies they would be expected to leak fluorescein, but experiments have shown that a fluid-filled space is necessary for this to occur. ${ }^{25}$ It is therefore possible that small vessels enveloped by pericytes as seen in case 1 may likewise lead to minimal leakage if there is little debris detaching the surrounding RPE, and fluorescein angiography performed on this patient 1 year before death had shown no evidence of CNV. It is also likely that the small new vessels commonly found on histological examination of senile eyes ${ }^{1,11}$ may similarly have failed to progress owing to a lack of diffuse debris. On the other hand, a more profuse deposition may contribute not only to hypoxia of the outer retina but also to loosening of the attachment of the RPE to BrM. The potential space thus created would allow the new vessel to leak fluid and fluorescein, as well as favouring its spread. This is akin to oedema of the cornea, which is a necessary prerequisite for the ingrowth of new vessels although it is not the sole factor involved. ${ }^{26}$

In case 2 the tips of the CNVM demonstrated morphological differences not only from the small new vessels in case 1 , but also around different parts of the membrane itself. Those tips, which leaked fibrin-like material, extended about $100 \mu \mathrm{m}$ beyond the main membrane, alternating with less leaky areas, and so would correspond to the scalloped edge commonly noted on fluorescein angiography. These tips showed an absence of pericytes but, in contrast to the experimental situations, the endothelial-lined channels were not bare and remained covered by a thick BsM-like material. Overlying the edge of the membrane the RPE exhibited hyperpigmentation and proliferation and it has been suggested that this accounts for the dark halo of blocked fluorescence commonly seen around CNVMs. $^{27}$

The examination of surgically removed CNVMs has provided correlation of their ultrastructure with the angiographic findings, ${ }^{9,27-29}$ but in many of these cases the vessels were already associated with fibrous tissue and there have been no descriptions of advancing tips lacking a pericyte cover. The present study suggests that certain assumptions can be made about the morphology and further progress of early $\mathrm{CNV}$ on the basis of fluorescein and indocyanine green (ICG) angiography. Profuse leakage of fluorescein from the advancing tips of a CNVM probably indicates that proteinaceous exudate is escaping, but more information might be provided by ICG which is $98 \%$ protein-bound, mostly to globulin. Since pericytes and their basal lamina appear to block extravasation of macromolecules in experimentally induced leakage, ${ }^{30}$ ICG fluorescence may point to the absence of pericytes. Moreover, escape of protein-bound ICG could correlate with the extravasation of fibrinogen and it is noteworthy that the fibrin in case 2 could be traced to the apex of the endothelial-lined vessels, without any indication that it was derived from the overlying neurosensory detachment as has been suggested. ${ }^{27}$ ICG fluorescence would therefore appear to be an unfavourable prognostic indicator.

The cases presented illustrate that the search for a means of modifying the neovascular form of ARM must take into consideration not only the factors inducing angiogenesis, but also those promoting further activation and spread of the new vessels. The change from a 'low-turnover' phase of angiogenesis to a 'high-turnover' phase appears to correlate with the amount of diffuse debris present in the sub-RPE space. The hypoxia that this induces in the outer retina may trigger an imbalance of angiogenic factors over inhibitory factors, while at the same time the debris creates a plane of least resistance favouring spread and leakage. Treatment modalities being explored therefore include attempts to confine the disease to the earlier 'low-turnover' phase, by applying photocoagulation in order to reduce the amount of soft drusen material and close the sub-RPE space.

This work was supported in part by the Retina Research Foundation, Houston, Texas, and the Gerontology Foundation of Australia.

Key words: Age-related macular degeneration, Choroidal neovascularisation, Electron microscopy, Pericytes.

\section{REFERENCES}

1. Sarks SH. New vessel formation beneath the retinal pigment epithelium in senile eyes. $\mathrm{Br} \mathrm{J}$ Ophthalmol 1973:57:951-65.

2. Green WR, McDonnell PJ, Yeo JH. Pathologic features of senile macular degeneration. Ophthalmology 1985;92:615-27.

3. Sarks SH. Ageing and degeneration in the macular region: a clinico-pathological study. $\mathrm{Br} \mathrm{J}$ Ophthalmol 1976;60:324-41.

4. Gregor Z, Bird AC, Chisholm IH. Senile disciform macular degeneration in the second eye. $\mathrm{Br} J$ Ophthalmol 1977;61:141-7.

5. Bressler SB, Macquarie MG, Bressler NM, Fine SL. The Macular Photocoagulation Study Group. Relationship of drusen and abnormalities of the retinal pigment epithelium to the prognosis of neovascular macular degeneration. Arch Ophthalmol 1990;108:1442-7. 
6. Killingsworth MC. Angiogenesis in early choroidal neovascularization secondary to age-related macular degeneration. Graefes Arch Clin Exp Ophthalmol 1995;233:313-23.

7. Killingsworth MC, Sarks JP, Sarks SH. Macrophages related to Bruch's membrane in age-related macular degeneration. Eye 1990;4:613-21.

8. Sarks JP, Sarks SH, Killingsworth MC. Evolution of soft drusen in age-related macular degeneration. Eye 1994;8:269-83.

9. Seregard S, Algvere PV, Berglin L. Immunohistochemical characterization of surgically removed subfoveal fibrovascular membranes. Graefes Arch Clin Exp Ophthalmol 1994;232:325-9.

10. Yamagami I. Electron microscopic study of the cornea. 1. The mechanism of experimental new vessel formation. Jpn J Ophthalmol 1970;14:41-58.

11. Green W, Enger C. Age-related macular degeneration: histopathologic studies. The 1992 Lorenz E. Zimmerman lecture. Ophthalmology 1993;100:1519-35.

12. Penfold PL. Senile macular degeneration: the involvement of immunocompetent cells. Graefes Arch Clin Exp Ophthalmol 1985;223:69-76.

13. Dastgheib K, Green WR. Granulomatous reaction to Bruch's membrane in age-related macular degeneration. Arch Ophthalmol 1994;112:813-8.

14. Shweiki D, Itin A, Soffer D, Keshet E. Vascular endothelial growth factor induced by hypoxia may mediate hypoxia-initiated angiogenesis. Nature 1992; 359:843-5.

15. Forrester JV, Chapman A, Kerr C, Roberts J, Lee WR, Lackie JM. Bovine retinal explants cultured in collagen gels: a model system for the study of proliferative retinopathy. Arch Ophthalmol 1990;108:415-20.

16. McLeod DS, Lutty GA. High-resolution histologic analysis of the human choroidal vasculature. Invest Ophthalmol Vis Sci 1994;35:3799-811.

17. Miller H, Miller B, Ishibashi T, Ryan SJ. Pathogenesis of laser-induced choroidal subretinal neovascularization. Invest Ophthalmol Vis Sci 1990;31:899-908.

18. Gass DJ. Biomicroscopic and histopathologic considerations regarding the feasibility of surgical excision of subfoveal neovascular membranes. Trans Am Ophthalmol Soc 1994;92:91-116.
19. Folkman J, Haudenschild C. Angiogenesis in vitro. Nature 1980;288:551-6.

20. Folkman J, Klagsbrun M. Angiogenic factors. Science 1987;235:442-7.

21. Ausprunk DH, Folkman J. Migration and proliferation of endothelial cells in preformed and newly formed blood vessels during tumor angiogenesis. Microvasc Res 1977;14:53-65.

22. Ishibashi T, Miller H, Orr G, Sorgente N, Ryan SJ. Morphologic observations on experimental subretinal neovascularization in the monkey. Invest Ophthalmol Vis Sci 1987;28:1116-30.

23. Ishibashi $\mathrm{T}$, Inomata $\mathrm{H}$, Sakamoto $\mathrm{T}$, Ryan $\mathrm{SJ}$. Pericytes of newly formed vessels in experimental subretinal neovascularization. Arch Ophthalmol 1995;113:227-31.

24. Orlidge-Antonelli A, Smith SR, D'Amore PA. Influence of pericytes on capillary endothelial cell growth. Am Rev Respir Dis 1989;140:1129-31.

25. Miller H, Miller B, Ryan SJ. Correlation of choroidal subretinal neovascularization with fluorescein angiography. Am J Ophthalmol 1985;99:263-71.

26. Maurice DM, Gaubermann H, Michaelson IC. Stimulus to neovascularisation in the cornea. Exp Eye Res 1966;5:163-84.

27. Lopez PF, Lambert HM, Grossniklaus HE, Sternberg P Jr. Well-defined subfoveal choroidal neovascular membranes in age-related macular degeneration. Ophthalmology 1993;100:415-22.

28. Lopez PF, Grossniklaus HE, Lambert HM, Aaberg TM, Capone A Jr, Sternberg P Jr, L'Hernault N. Pathologic features of surgically excised subretinal neovascular membranes in age-related macular degeneration. Am J Ophthalmol 1991;112:647-56.

29. Lee BL, Lim JI, Grossniklaus HE. Clinicopathologic features of indocyanine green angiography-imaged, surgically excised choroidal neovascular membranes. Retina 1996;16:64-9.

30. Sims DE, Miller F, Perricone MA. A role of pericytes in skeletal muscle vasculature during macromolecular leakage. Microvasc Res 1985;29:250. 